\title{
Mixed Rational Lump-Solitary Wave Solutions to an Extended (2+1)-Dimensional KdV Equation
}

\author{
Zhigang Yao, ${ }^{1,2}$ Huayong Xie $\mathbb{D}^{3},{ }^{3}$ and Hui Jie ${ }^{1}$ \\ ${ }^{1}$ Department of Electronic and Optic Engineering, Army Engineering University, Shijiazhuang 050003, China \\ ${ }^{2}$ School of Automation and Electrical Engineering, University of Science and Technology, Beijing 100083, China \\ ${ }^{3}$ Faculty of Preschool Teacher Education, Lishui University, Lishui 323000, China
}

Correspondence should be addressed to Huayong Xie; huayongxie@lsu.edu.cn

Received 26 January 2021; Revised 27 February 2021; Accepted 7 March 2021; Published 23 March 2021

Academic Editor: Laurent Raymond

Copyright (C) 2021 Zhigang Yao et al. This is an open access article distributed under the Creative Commons Attribution License, which permits unrestricted use, distribution, and reproduction in any medium, provided the original work is properly cited.

Based on the bilinear method, rational lump and mixed lump-solitary wave solutions to an extended $(2+1)$-dimensional KdV equation are constructed through the different assumptions of the auxiliary function in the trilinear form. It is found that the rational lump decays algebraically in all directions in the space plane and its amplitude possesses one maximum and two minima. One kind of the mixed solution describes the interaction between one lump and one line solitary wave, which exhibits fission and fusion phenomena under the different parameters. The other kind of the mixed solution shows one lump interacting with two paralleled line solitary waves, in which the evolution of the lump gives rise to a two-dimensional rogue wave. This shows that these three interesting phenomena exist in the corresponding physical model.

\section{Introduction}

The study of integrable nonlinear systems has become a hot topic in wave propagations and mathematical physics. Integrable systems approximately describe the evolution of various waves in many physical settings, including shallowwater waves with weakly nonlinear restoring forces, pulse propagation in optical fibers and wave guides, long internal waves in a density-stratified ocean, and ion acoustic waves in plasma [1-16]. In the higher-dimensional extensions of integrable nonlinear wave equation, the $(2+1)$-dimensional KdV equation or the asymmetrical Nizhnik-NovikovVeselov (ANNV) equation [17]

$$
u_{t}+u_{x x x}+3\left(u \partial_{y}^{-1} u_{x}\right)_{x}=0
$$

was firstly proposed by Boiti et al. in the sense of the weak Lax pair. This model arose in the incompressible fluid and was shown to possess an infinite number of conservation laws, multiple soliton solutions, and other integrability properties [17]. By introducing two terms $\partial_{y}^{-1} u_{x x}$ and $u_{y}=\partial_{y}^{-1} u_{y y}$ into equation (1), a generalized (2+1)-dimensional $\mathrm{KdV}$ equation with arbitrary constant coefficients has been recently developed [18]

$$
u_{t}+v_{x x x}+\alpha\left(u \partial_{y}^{-1} u_{x}\right)_{x}+\beta\left(\partial_{y}^{-1} u_{x x}\right)+\gamma\left(\partial_{y}^{-1} u_{y y}\right)=0,
$$

which describes the ion-acoustic waves in plasmas, shallow water waves in oceans, and pulse waves in large arteries. Here, $\alpha, \beta$, and $\gamma$ are real constants. Equation (2) reduces to the ANNV equation (1) when $\alpha=3$ and $\beta=\gamma=0$ and becomes the classical KdV equation when $y=x$. The $(2+1)$ dimensional equation (2) was investigated through the Painlevé test, and its multiple-soliton solutions were derived via the simplified Hirota algorithm [18]. More recently, a lot of rational lump solutions, hybrid solutions consisting of lump waves and kink waves, loop-like kink breather solutions, and the lump interacting with the line soliton solutions have been constructed via the Hirota bilinear method [19-46]. Although these results can also be derived by Darboux transformation [47-50], modified extended mapping method [1], and direct algebraic method [2], the bilinear method is still a powerful tool for solving integrable systems. It is worth 
mentioning that Seadawy et al. obtained some new exact solutions of many integrable systems by using various methods, such as extend simple equation method and the $\exp (\phi(\xi))$ expansion method [1-16]. For the ANNV equation (1), the lump solutions, mixed lump-stripe solutions, and periodic lump solutions were presented in [19].

Recently, researches about trilinear form have become a hot topic. Trilinear form is an extension of Hirota's bilinear form [51]. A group of scholars who work on integrable systems have found that some new analytic solutions of nonlinear PDEs can be obtained through trilinear differential equations $[52,53]$. Hence, we aim to construct the rational lump and the lump-solitary wave solutions to the extended $(2+1)$-dimensional $\mathrm{KdV}$ equation (2) through the trilinear form.

The rest of the paper is organized as follows. In Section 2, we firstly transform the extended $(2+1)$-dimensional $\mathrm{KdV}$ equation (2) to the trilinear form through the certain variable transformation and construct the exact rational lump solution. The mixed solution composed of one lump and one line solitary wave is derived in Section 3. Section 4 devotes to studying the interaction solution consisting of one lump and two line solitary waves, which can be viewed as a twodimensional rogue wave excited from the line soliton pair.

\section{Lump Solution}

Through the dependent variable transformation $u=(6 / \alpha)$ $(\ln f)_{x y}$, the extended $(2+1)$-dimensional $\mathrm{KdV}$ equation (2) is transformed to the following trilinear form:

$$
\begin{aligned}
{\left[f, B_{1} f \cdot f\right]_{y} } & +\beta\left[f, D_{x}^{2} f \cdot f\right]_{x}-3\left[f_{x x}, D_{x} D_{y} f \cdot f\right]_{x} \\
& +3\left[f_{x x}, D_{x}^{2} f \cdot f\right]_{y}=0
\end{aligned}
$$

where

$$
\begin{gathered}
B_{1} f \cdot f=D_{x} D_{t} f \cdot f+D_{x}^{4} f \cdot f+\gamma D_{x} D_{y} f \cdot f, \\
{[a, b]_{x}=a_{x} b-\frac{1}{2} a b_{x},} \\
{[a, b]_{y}=a_{y} b-\frac{1}{2} a b_{y},}
\end{gathered}
$$

and the Hirota bilinear operators $D_{x}, D_{y}$, and $D_{t}$ are defined by [54]

$$
\begin{aligned}
D_{x}^{n} D_{y}^{m} D_{t}^{l}(a \cdot b)= & \left(\frac{\partial}{\partial x}-\frac{\partial}{\partial x^{\prime}}\right)^{n}\left(\frac{\partial}{\partial y}-\frac{\partial}{\partial y^{\prime}}\right)^{m}\left(\frac{\partial}{\partial t}-\frac{\partial}{\partial t^{\prime}}\right)^{l} \\
& \cdot a(x, y, t) b x^{\prime}, y^{\prime},\left.t^{\prime}\right|_{x^{\prime}=x, y^{\prime}=y, t^{\prime}=t} .
\end{aligned}
$$

In order to find the rational lump solution for equation (2), we set the auxiliary variable $f$ in equation (3) as the following form:

$$
\begin{gathered}
f=g^{2}+h^{2}+a_{9}, \\
g=a_{1} x+a_{2} y+a_{3} t+a_{4}, \\
h=a_{5} x+a_{6} y+a_{7} t+a_{8},
\end{gathered}
$$

with

$$
\left|\begin{array}{ll}
a_{1} & a_{2} \\
a_{5} & a_{6}
\end{array}\right| \neq 0
$$

where $a_{i}(i=1,2, \cdots, 9)$ are real parameters and will be determined. Based on symbolic computation, we substitute the assumption equation (6) into the trilinear equation (3) and then collect the coefficients of the independent variables $x, y$, and $t$. Consequently, one has a set of algebraic equations with respect to the parameters $a_{i}(i=1,2, \cdots, 9)$. To solve these equations, one can get the parameter relations as follows:

$$
\begin{aligned}
& a_{3}=-\frac{\beta\left[a_{2}\left(a_{1}^{2}-a_{5}^{2}\right)+2 a_{1} a_{5} a_{6}\right]}{a_{2}^{2}+a_{6}^{2}}-\gamma a_{2}, \\
& a_{7}=-\frac{\beta\left[a_{6}\left(a_{5}^{2}-a_{1}^{2}\right)+2 a_{1} a_{2} a_{5}\right]}{a_{2}^{2}+a_{6}^{2}}-\gamma a_{6}, \\
& a_{9}=-\frac{3\left(a_{1}^{2}+a_{5}^{2}\right)\left(a_{2}^{2}+a_{6}^{2}\right)\left(a_{1} a_{2}+a_{5} a_{6}\right) .}{\beta\left(a_{1} a_{6}-a_{2} a_{5}\right)^{2}} .
\end{aligned}
$$

This in turn gives rise to the rational lump solution as

$$
u=\frac{12\left(a_{1} a_{2}+a_{5} a_{6}\right)}{\alpha\left(g^{2}+h^{2}+a_{9}\right)}-\frac{24\left(a_{1} g+a_{5} h\right)\left(a_{2} g+a_{6} h\right)}{\alpha\left(g^{2}+h^{2}+a_{9}\right)^{2}},
$$

where the functions $g$ and $h$ are given in equation (6) with the parameters' conditions (8) and (9). To guarantee that the function $f$ is well defined and the solution $u$ in equation (10) decays in all directions in the $x, y$ plane, these parameters are restricted by three conditions: $a_{2}^{2}+a_{6}^{2} \neq 0$ and $-\left(\left(3\left(a_{1}^{2}+a_{5}^{2}\right)(\right.\right.$ $\left.\left.\left.a_{1} a_{2}+a_{5} a_{6}\right)\right) / \beta\right)>0$.

For the local analysis, we find that the rational lump solution $u$ in equation (10) possesses the maximum amplitude $-\left(\left(4 \beta\left(a_{1} a_{6}-a_{2} a_{5}\right)^{2}\right) /\left(\alpha\left(a_{1}^{2}+a_{5}^{2}\right)\left(a_{2}^{2}+a_{6}^{2}\right)\right)\right)$, which is centered at the point

$$
\left(\frac{a_{2} a_{8}-a_{4} a_{6}+\left(a_{2} a_{7}-a_{3} a_{6}\right) t}{a_{1} a_{6}-a_{2} a_{5}}, \frac{a_{4} a_{5}-a_{1} a_{8}+\left(a_{3} a_{5}-a_{1} a_{7}\right) t}{a_{1} a_{6}-a_{2} a_{5}}\right) .
$$

It is concluded that this rational lump moves along the route line $l_{0}: y=\left(\left(a_{3} a_{5}-a_{1} a_{7}\right) /\left(a_{2} a_{7}-a_{3} a_{6}\right)\right) x+\left(\left(a_{3} a_{8}-\right.\right.$ $\left.\left.a_{4} a_{7}\right) /\left(a_{2} a_{7}-a_{3} a_{6}\right)\right)$ and with the velocities $V_{x}=\left(a_{2} a_{7}-a_{3}\right.$ $\left.a_{6}\right) /\left(a_{1} a_{6}-a_{2} a_{5}\right)$ and $V_{y}=\left(a_{3} a_{5}-a_{1} a_{7}\right) /\left(a_{1} a_{6}-a_{2} a_{5}\right)$, respectively.

The specific lump's structure and its moving path are illustrated in Figure 1. With the given parameter's values, 

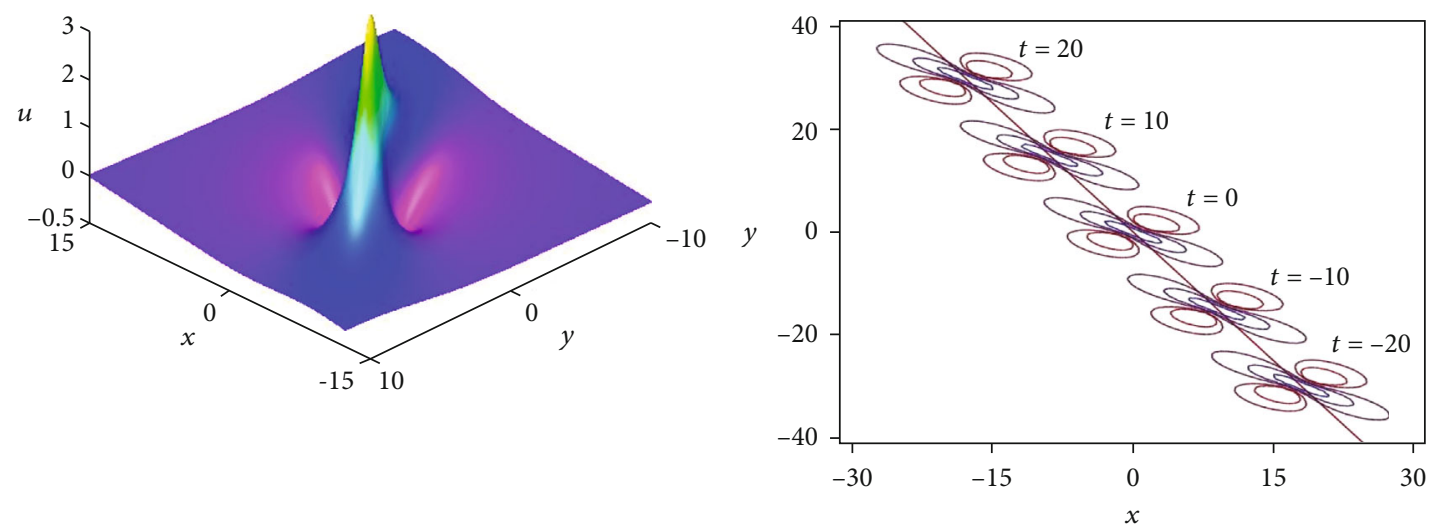

(a)

(b)

FIGURE 1: The rational lump solution equation (10) with $a_{1}=a_{5}=\alpha=-\beta=\gamma=1, a_{2}=-1 / 5, a_{6}=2$, and $a_{4}=a_{8}=0$ : (a) the three-dimensional plot at $t=0$; (b) the moving path with the contour curves of lumps at the different times.

Figure 1(a) shows the three-dimensional plot of the rational lump equation (10) at time $t=0$, and Figure 1(b) corresponds to the contour curves at the different time, which exhibits the moving track of the lump. The direct calculation indicates that the illustrated lump in Figure 1 moves along the straight line $l_{0}: y=-(151 / 90) x$, its velocities are $V_{x}=-(90 / 101), V_{y}=151 / 101$, and its maximum amplitude is $242 / 101$.

\section{The Mixed Solution Composed of One Rational Lump and One Line Solitary Wave}

To construct the mixed solution that is composed of one rational lump and one line solitary wave, we use the following assumption of the function $f$ :

$$
\begin{gathered}
f=g^{2}+h^{2}+a_{9}+k e^{\eta}, \\
g=a_{1} x+a_{2} y+a_{3} t+a_{4}, \\
h=a_{5} x+a_{6} y+a_{7} t+a_{8}, \\
\eta=k_{1} x+k_{2} y+k_{3} t,
\end{gathered}
$$

where $a_{i}(i=1,2, \cdots, 9), k$, and $k_{i}(i=1,2,3)$ are real parameters and will be determined. Here, the rational and the exponential functions are responsible for the rational lump and the line solitary wave, respectively. Similar to the case of the purely rational lump, one needs to collect the coefficients of $x, y$, and $t$ and exponential functions after substituting equation (12) into equation (3). Then, we have a set of algebraic equations with respect to the parameters $a_{i}(i=1,2, \cdots, 9), k$, and $k_{i}(i=1,2,3)$, which gives the parameters' relations as follows:

$$
\begin{aligned}
& a_{3}=-\frac{\beta\left[a_{2}\left(a_{1}^{2}-a_{5}^{2}\right)+2 a_{1} a_{5} a_{6}\right]}{a_{2}^{2}+a_{6}^{2}}-\gamma a_{2}, \\
& a_{7}=-\frac{\beta\left[a_{6}\left(a_{5}^{2}-a_{1}^{2}\right)+2 a_{1} a_{2} a_{5}\right]}{a_{2}^{2}+a_{6}^{2}}-\gamma a_{6},
\end{aligned}
$$

$$
\begin{gathered}
a_{9}=-\frac{3\left(a_{1}^{2}+a_{5}^{2}\right)\left(a_{2}^{2}+a_{6}^{2}\right)\left(a_{1} a_{2}+a_{5} a_{6}\right)}{\beta\left(a_{1} a_{6}-a_{2} a_{5}\right)^{2}}, \\
k_{3}=-k_{1}^{3}-\beta \frac{k_{1}^{2}}{k_{2}}-\gamma k_{2}, \\
k_{1}=\frac{a_{1} a_{2}+a_{5} a_{6}}{a_{2}^{2}+a_{6}^{2}} k_{2}-\frac{3\left(a_{1}^{2}+a_{5}^{2}\right)}{2 \beta\left(a_{2}^{2}+a_{6}^{2}\right)} k_{2}^{3}, \\
k_{2}=\frac{\delta_{1}}{3}\left[\frac{6 \beta\left(a_{1} a_{2}+a_{5} a_{6}\right)}{a_{1}^{2}+a_{5}^{2}}+6 \sigma_{2} \beta\left(\frac{a_{2}^{2}+a_{6}^{2}}{a_{1}^{2}+a_{5}^{2}}\right)^{1 / 2}\right]^{1 / 2},
\end{gathered}
$$

with $\sigma_{i}^{2}=1$ for $i=1,2$. This in turn leads to the mixed solution composed of one lump and one line solitary wave as

$$
\begin{aligned}
u= & \frac{6\left(2 a_{1} a_{2}+2 a_{5} a_{6}+k k_{1} k_{2} e^{\eta}\right)}{\alpha\left(g^{2}+h^{2}+a_{9}+k e^{\eta}\right)} \\
& -\frac{6\left(2 a_{1} g+2 a_{5} h+k k_{1} e^{\eta}\right)\left(2 a_{2} g+2 a_{6} h+k k_{2} e^{\eta}\right)}{\alpha\left(g^{2}+h^{2}+a_{9}+k e^{\eta}\right)^{2}},
\end{aligned}
$$

where

$a_{1} a_{6}-a_{2} a_{5} \neq 0,2_{2}+a_{6}^{2} \neq 0,-\frac{3\left(a_{1}^{2}+a_{5}^{2}\right)\left(a_{1} a_{2}+a_{5} a_{6}\right)}{\beta}>0, k>0$.

Here, $g, h$, and $\eta$ are defined by equation (12) with the parameters' relations (13), (14), and (15). The restricted conditions in equation (16) are to be able to form a lump wave and guarantee the regularity of the function $f$.

In the interaction processes between one lump and one line solitary wave, fission and fusion phenomena $[55,56]$ will appear under the different parameters. If we set $x$ and $y$ as constants, the structure of the mixed solution equation (16) can be explained as follows. When the coefficient of the time $k_{3}>0$, the exponential term is dominant and only the line solitary wave exists for $t>0$, while the 


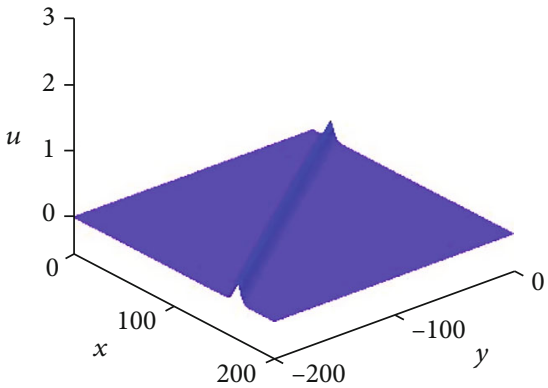

(a)

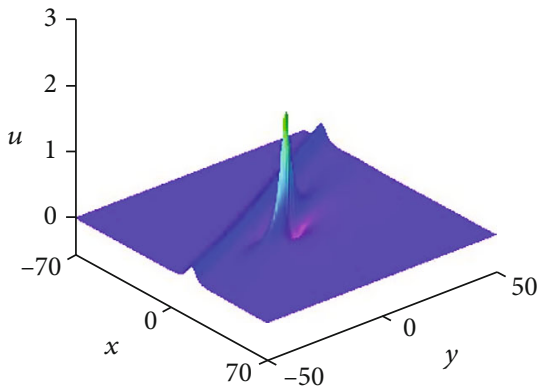

(c)

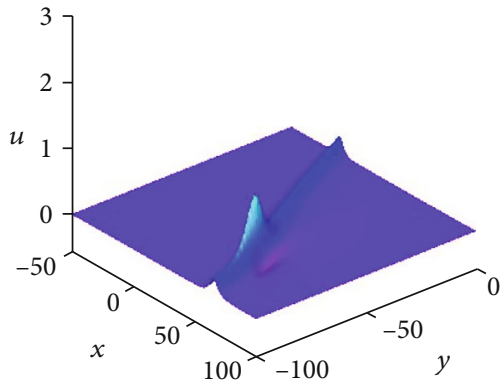

(b)

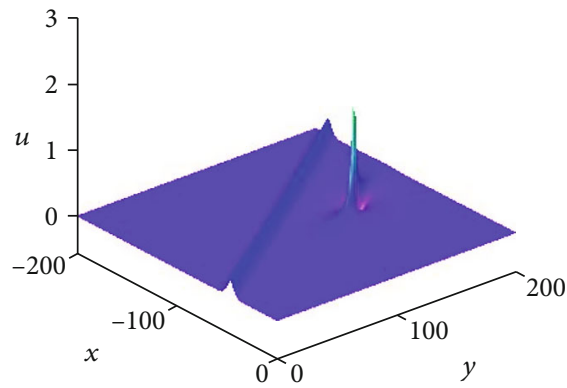

(d)

Figure 2: The three-dimensional plots of the mixed solution equation (16) with $a_{1}=1 / 20, a_{5}=2, k=1 / 10, a_{2}=a_{6}=\alpha=-\beta=\gamma=-\sigma_{1}=-$ $\sigma_{2}=1$, and $a_{4}=a_{8}=0$ : (a) $t=-100$; (b) $t=-25$; (c) $t=0$; (d) $t=50$.

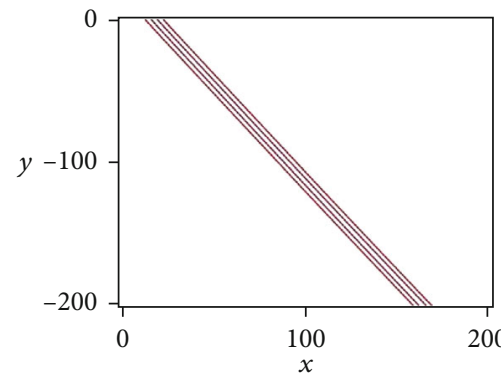

(a)

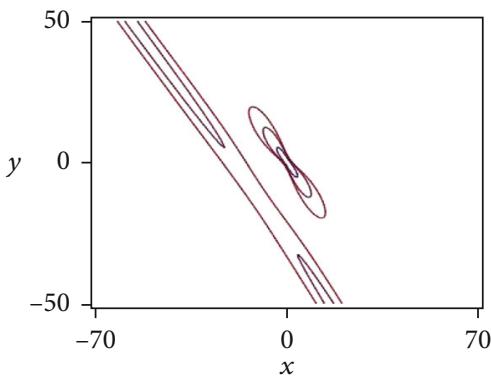

(c)

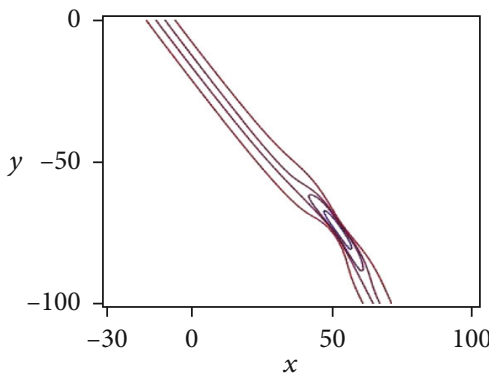

(b)

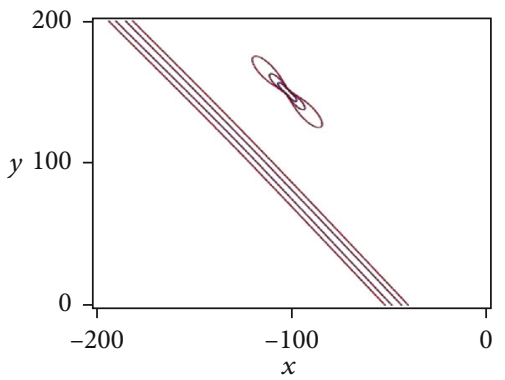

(d)

Figure 3: The corresponding contour plots of the mixed solution equation (16) with $a_{1}=1 / 20, a_{5}=2, k=1 / 10, a_{2}=a_{6}=\alpha=-\beta=\gamma=-\sigma_{1}$ $=-\sigma_{2}=1$, and $a_{4}=a_{8}=0$ : (a) $t=-100$; (b) $t=-25$; (c) $t=0$; (d) $t=50$.

rational term is dominant and the rational lump emerges for $t<0$. Thus, such interaction processes correspond to a fission phenomenon. On the contrary, the negative coefficient of the time $k_{3}$ gives rise to a fusion phenomenon. To illustrate this type of the mixed solution, we exhibit the fission phenomenon through the three-dimensional plots in Figure 2 and the corresponding contour plots in Figure 3. It can be seen clearly that only one line solitary wave exists firstly and then one rational lump arises gradually. Although the integrable system studied in this paper is not the same system as those in Refs. [55, 56], they have similar fusion and fission phenomena. 


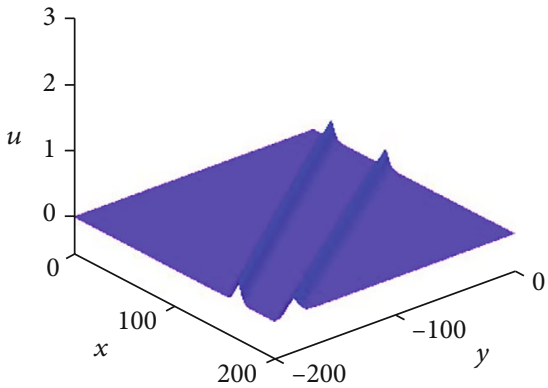

(a)

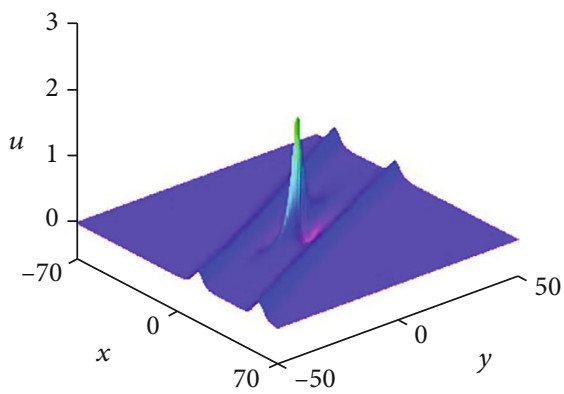

(c)

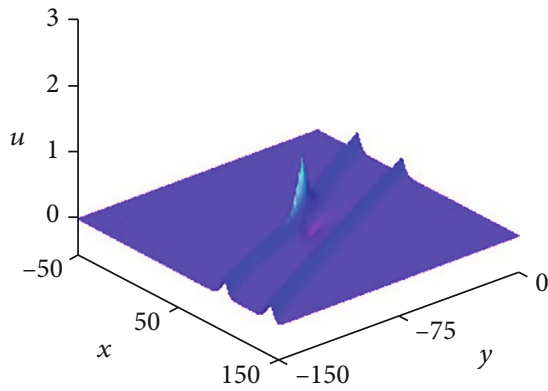

(b)

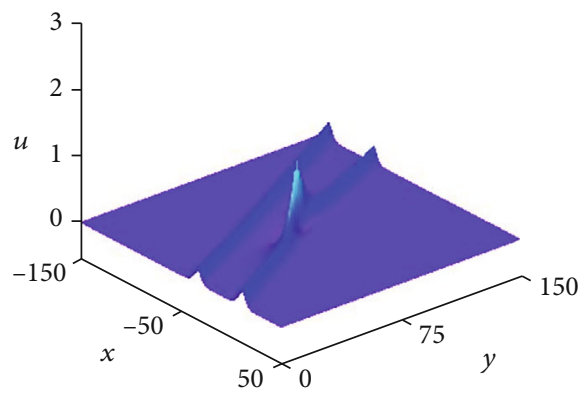

(d)

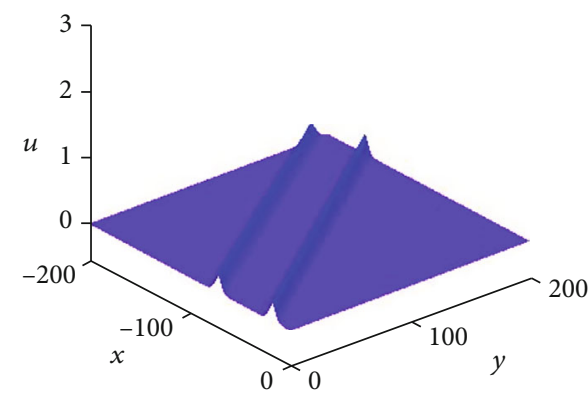

(e)

FIgURE 4: The three-dimensional plots of the mixed solution equation (23) with $a_{1}=1 / 20, a_{5}=2, k=l=1 / 10, a_{2}=a_{6}=\alpha=-\beta=\gamma=-\sigma_{1}=$ $-\sigma_{2}=1$, and $a_{4}=a_{8}=0$ : (a) $t=-100$; (b) $t=-25$; (c) $t=0$; (d) $t=25$; (e) $t=100$.

\section{The Mixed Solution Composed of One Rational Lump and Two Line Solitary Waves}

In this section, we seek to construct the mixed solution composed of one rational lump and two line solitary waves. This type of interaction solution will describe fission and fusion phenomena simultaneously. According to the last section, we need to assume the function $f$ as the following form:

$$
\begin{gathered}
f=g^{2}+h^{2}+a_{9}+k e^{\eta}+l e^{-\eta}, \\
g=a_{1} x+a_{2} y+a_{3} t+a_{4}, \\
h=a_{5} x+a_{6} y+a_{7} t+a_{8}, \\
\eta=k_{1} x+k_{2} y+k_{3} t,
\end{gathered}
$$

where $a_{i}(i=1,2, \cdots, 9), k, l$, and $k_{i}(i=1,2,3)$ are real parameters and will be determined. Here, the rational and the exponential terms support the rational lump and the line soliton pair, respectively. Proceeding as before, we have the parameters' relations as follows:

$$
\begin{aligned}
& a_{3}=-\frac{\beta\left[a_{2}\left(a_{1}^{2}-a_{5}^{2}\right)+2 a_{1} a_{5} a_{6}\right]}{a_{2}^{2}+a_{6}^{2}}-\gamma a_{2}, \\
& a_{7}=-\frac{\beta\left[a_{6}\left(a_{5}^{2}-a_{1}^{2}\right)+2 a_{1} a_{2} a_{5}\right]}{a_{2}^{2}+a_{6}^{2}}-\gamma a_{6},
\end{aligned}
$$

$$
\begin{aligned}
a_{9}= & -\frac{3\left(a_{1}^{2}+a_{5}^{2}\right)\left(a_{2}^{2}+a_{6}^{2}\right)\left(a_{1} a_{2}+a_{5} a_{6}\right)}{\beta\left(a_{1} a_{6}-a_{2} a_{5}\right)^{2}} \\
& +\frac{8 \beta k l\left[\beta\left(a_{1} a_{6}-a_{2} a_{5}\right)^{2}+3 k_{2}^{2}\left(a_{1}^{2}+a_{5}^{2}\right)\left(a_{1} a_{2}+a_{5} a_{6}\right)\right]}{3\left(a_{1} a_{6}-a_{2} a_{5}\right)^{2}\left[3 k_{2}^{2}\left(a_{1}^{2}+a_{5}^{2}\right)-2 \beta\left(a_{1} a_{2}+a_{5} a_{6}\right)\right]},
\end{aligned}
$$




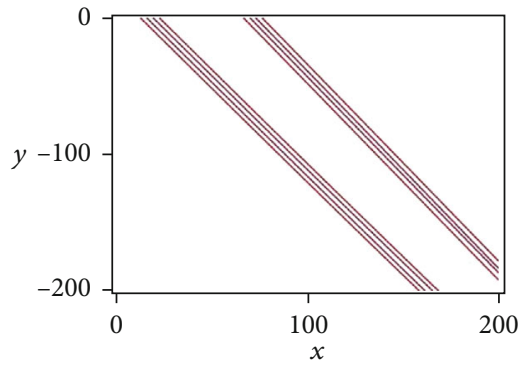

(a)

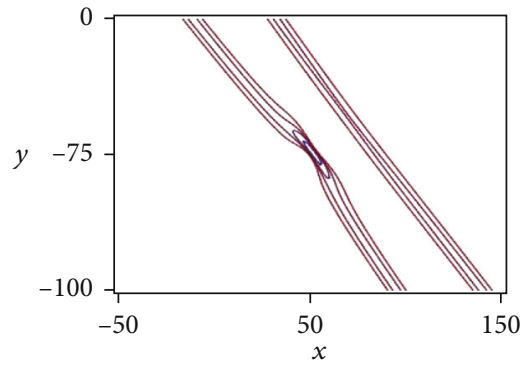

(b)

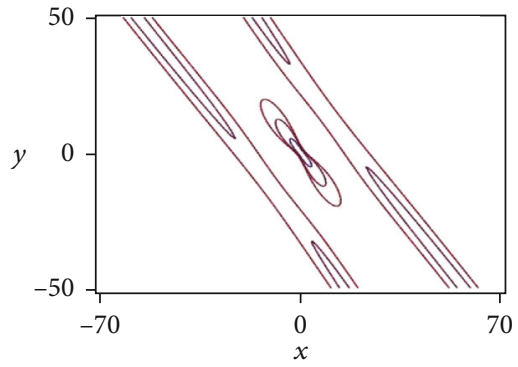

(c)

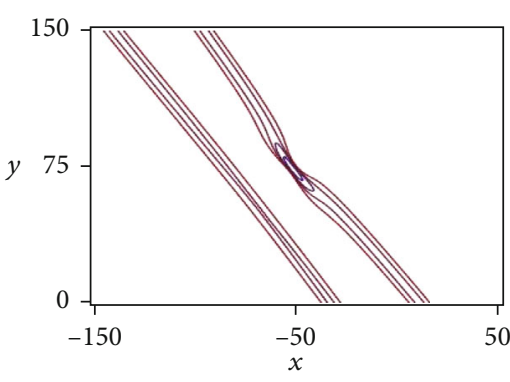

(d)

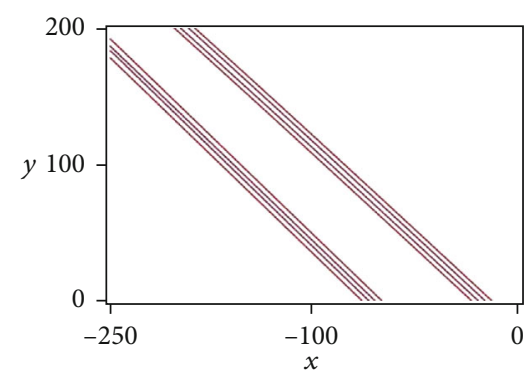

(e)

FIGURE 5: The corresponding contour plots of the mixed solution equation (23) with $a_{1}=1 / 20, a_{5}=2, k=l=1 / 10, a_{2}=a_{6}=\alpha=-\beta=\gamma=-$ $\sigma_{1}=-\sigma_{2}=1$, and $a_{4}=a_{8}=0$ : (a) $t=-100$; (b) $t=-25$; (c) $t=0$; (d) $t=25$; (e) $t=100$.

$$
\begin{gathered}
k_{3}=-k_{1}^{3}-\beta \frac{k_{1}^{2}}{k_{2}}-\gamma k_{2}, \\
k_{1}=\frac{a_{1} a_{2}+a_{5} a_{6}}{a_{2}^{2}+a_{6}^{2}} k_{2}-\frac{3\left(a_{1}^{2}+a_{5}^{2}\right)}{2 \beta\left(a_{2}^{2}+a_{6}^{2}\right)} k_{2}^{3}, \\
k_{2}=\frac{\delta_{1}}{3}\left[\frac{6 \beta\left(a_{1} a_{2}+a_{5} a_{6}\right)}{a_{1}^{2}+a_{5}^{2}}+6 \sigma_{2} \beta\left(\frac{a_{2}^{2}+a_{6}^{2}}{a_{1}^{2}+a_{5}^{2}}\right)^{1 / 2}\right]^{1 / 2},
\end{gathered}
$$

with $\sigma_{i}^{2}=1$ for $i=1,2$. This in turn gives the mixed solution composed of one lump and two line solitary waves as

$$
\begin{aligned}
u= & \frac{6\left[2 a_{1} a_{2}+2 a_{5} a_{6}+k_{1} k_{2}\left(k e^{\eta}+l e^{-\eta}\right)\right]}{\alpha\left(g^{2}+h^{2}+a_{9}+k e^{\eta}+l e^{-\eta}\right)} \\
& -\frac{6\left[2 a_{1} g+2 a_{5} h+k_{1}\left(k e^{\eta}-l e^{-\eta}\right)\right]\left[2 a_{2} g+2 a_{6} h+k_{2}\left(k e^{\eta}-l e^{-\eta}\right)\right]}{\alpha\left(g^{2}+h^{2}+a_{9}+k e^{\eta}+l e^{-\eta}\right)^{2}},
\end{aligned}
$$

with

$$
a_{1} a_{6}-a_{2} a_{5} \neq 0, a_{2}^{2}+a_{6}^{2} \neq 0, \quad a_{9}>0, k>0, l>0 .
$$

Here, $g, h$, and $\eta$ are defined by equation (18) with the parameters' relations (19), (20), (21), and (22). In this interaction processes described by equation (23), both fission and fusion phenomena will occur under the certain parameters' values. Thus, it can be realized that the lump is only observed on a certain region or during a specific time period. More precisely, by setting $x$ and $y$ as the fixed constants in the mixed solution equation (18), one can give the simple analysis:

$$
\begin{gathered}
\lim _{t \longrightarrow \pm \infty} \frac{g^{2}}{h^{2}}=\frac{a_{3}^{2}}{a_{7}^{2}}, \\
\lim _{t \longrightarrow \pm \infty} \frac{g^{2}}{k e^{\eta}+l e^{-\eta}}=0, \\
\lim _{t \longrightarrow \pm \infty} \frac{h^{2}}{k e^{\eta}+l e^{-\eta}}=0, \\
(k, l>0) .
\end{gathered}
$$

It implies that only two line solitary waves exist when the time approaches to infinity, and the lump emerges and reaches its maximum amplitude when the time approaches to zero. Hence, the evolution of the lump coincides with the characters of rogue wave: short-lived occurrence and large amplitude. The rational lump is identified as a two-dimensional rogue wave originating in the line soliton pair. The threedimensional plots and corresponding contour plots for this type of the mixed solution at different times are shown in Figures 4 and 5, respectively. It can be observed that in the evolution process the lump acts as a rogue wave but the line soliton pair remains the same shape. The whole interaction means that a two-dimensional rogue wave is excited from two paralleled line solitary waves.

\section{Conclusions}

In this paper, we have constructed rational lump and mixed lump-solitary wave solutions of the extended $(2+1)$-dimensional $\mathrm{KdV}$ equation by using the bilinear method. Under the appropriate variable transformation, the extended $(2+1)$-dimensional $\mathrm{KdV}$ equation is firstly changed into the 
trilinear form. Then, three groups of exact solutions are derived by assuming the auxiliary function as the quadratic and exponential functions. The first kind of solution is given by the purely rational form, it possesses one maximum and two minima, and its peak decays algebraically in all directions in the space plane. Figure 1 shows these characteristics of a lump wave intuitively and clearly. The second kind of solution is expressed by the mixed rational-exponential function, which exhibits fission and fusion phenomena between one lump and one line solitary wave. Equation (16) gives specific mathematical expressions for the second type of solution, and Figures 2 and 3 elaborate on these interesting fission and fusion phenomena. The last one contains one lump and two line solitary waves; these local waves' interaction shown in Figure 5 is able to describe a two-dimensional rogue wave excited from the line soliton pair. Because the extended (2+1)-dimensional KdV equation describes the ion-acoustic waves in plasmas, shallow water waves in oceans, and pulse waves in large arteries, we believe that there are fission and fusion phenomena in corresponding physical models.

\section{Data Availability}

No data were used to support this study.

\section{Conflicts of Interest}

The authors declare that they have no conflicts of interest.

\section{Acknowledgments}

This work was supported by Foundation Enhancement Programme Fund (2019-JCJQ-JJ-012).

\section{References}

[1] M. Arshad, A. R. Seadawy, and D. Lu, "Modulation stability and optical soliton solutions of nonlinear Schrodinger equation with higher order dispersion and nonlinear terms and its applications," Superlattices and Microstructures, vol. 112, pp. 422-434, 2017.

[2] M. Arshad, A. R. Seadawy, and D. Lu, "Elliptic function and solitary wave solutions of the higher-order nonlinear Schrödinger dynamical equation with fourth-order dispersion and cubic-quintic nonlinearity and its stability," The European Physical Journal Plus, vol. 132, no. 8, p. 371, 2017.

[3] D. Lu, A. R. Seadawy, and A. Ali, "Applications of exact traveling wave solutions of modified Liouville and the symmetric regularized long wave equations via two new techniques," Results in Physics, vol. 9, pp. 1403-1410, 2018.

[4] M. A. Helal, A. R. Seadawy, and M. H. Zekry, "Stability analysis of solitary wave solutions for the fourth-order nonlinear Boussinesq water wave equation," Applied Mathematics and Computation, vol. 232, pp. 1094-1103, 2014.

[5] D. Lu, A. R. Seadawy, and A. Ali, "Dispersive traveling wave solutions of the equal-width and modified equal-width equations via mathematical methods and its applications," Results in Physics, vol. 9, pp. 313-320, 2018.

[6] A. R. Seadawy, "Two-dimensional interaction of a shear flow with a free surface in a stratified fluid and its solitary-wave solutions via mathematical methods," The European Physical Journal Plus, vol. 132, no. 12, p. 518, 2017.

[7] A. Ali, A. R. Seadawy, and D. Lu, "Computational methods and traveling wave solutions for the fourth-order nonlinear Ablowitz-Kaup-Newell-Segur water wave dynamical equation via two methods and its applications," Open Physics, vol. 16, no. 1, pp. 219-226, 2018.

[8] M. Iqbal, A. R. Seadawy, O. H. Khalil, and D. Lu, "Propagation of long internal waves in density stratified ocean for the $(2+1)$ dimensional nonlinear Nizhnik-Novikov-Vesselov dynamical equation," Results in Physics, vol. 16, article 102838, 2020.

[9] A. R. Seadawy, M. Iqbal, and D. Lu, "Nonlinear wave solutions of the Kudryashov-Sinelshchikov dynamical equation in mixtures liquid-gas bubbles under the consideration of heat transfer and viscosity," Journal of Taibah University for Science, vol. 13, no. 1, pp. 1060-1072, 2019.

[10] N. Cheemaa, A. R. Seadawy, and S. Chen, "More general families of exact solitary wave solutions of the nonlinear Schrödinger equation with their applications in nonlinear optics," The European Physical Journal Plus, vol. 133, no. 12, p. 547, 2018.

[11] N. Cheemaa, A. R. Seadawy, and S. Chen, "Some new families of solitary wave solutions of the generalized Schamel equation and their applications in plasma physics," The European Physical Journal Plus, vol. 134, no. 3, p. 117, 2019.

[12] A. R. Seadawy and N. Cheemaa, "Propagation of nonlinear complex waves for the coupled nonlinear Schrodinger equations in two core optical fibers," Physica A, vol. 529, article 121330, 2019.

[13] N. Cheemaa, S. Chen, and A. R. Seadawy, "Propagation of isolated waves of coupled nonlinear $(2+1)$-dimensional Maccari system in plasma physics," Results in Physics, vol. 17, article 102987, 2020.

[14] H. Ahmad, A. R. Seadawy, T. A. Khan, and P. Thounthong, "Analytic approximate solutions for some nonlinear parabolic dynamical wave equations," Journal of Taibah University for Science, vol. 14, no. 1, pp. 346-358, 2020.

[15] H. Ahmad, A. R. Seadawy, and T. A. Khan, "Study on numerical solution of dispersive water wave phenomena by using a reliable modification of variational iteration algorithm," Mathematics and Computers in Simulation, vol. 177, pp. 13-23, 2020.

[16] N. Farah, A. R. Seadawy, S. Ahmad, S. T. R. Rizvi, and M. Younis, "Interaction properties of soliton molecules and Painleve analysis for nano bioelectronics transmission model," Optical and Quantum Electronics, vol. 52, no. 7, p. 329, 2020.

[17] M. Boiti, J. Leon, M. Manna, and F. Pempinelli, "On the spectral transform of a Korteweg-de Vries equation in two spatial dimensions," Inverse Problems, vol. 2, no. 3, pp. 271-279, 1986.

[18] A. M. Wazwaz, "Two new Painleve-integrable $(2+1)$ and (3+1)-dimensional KdV equations with constant and timedependent coefficients," Nuclear Physics B, vol. 954, article $115009,2020$.

[19] Z. L. Zhao, Y. Chen, and B. Han, "Lump soliton, mixed lump stripe and periodic lump solutions of a $(2+1)$-dimensional asymmetrical Nizhnik Novikov Veselov equation," Modern Physics Letters B, vol. 31, no. 14, article 1750157, 2017.

[20] W. X. Ma, "Lump solutions to the Kadomtsev-Petviashvili equation," Physics Letters A, vol. 379, no. 36, pp. 1975-1978, 2015.

[21] X. E. Zhang and Y. Chen, "Rogue wave and a pair of resonance stripe solitons to a reduced (3+1)-dimensional Jimbo-Miwa 
equation," Communications in Nonlinear Science and Numerical Simulation, vol. 52, pp. 24-31, 2017.

[22] X. E. Zhang and Y. Chen, "Deformation rogue wave to the (2+1)-dimensional KdV equation," Nonlinear Dynamics, vol. 90, no. 2, pp. 755-763, 2017.

[23] X. E. Zhang, Y. Chen, and X. Y. Tang, "Rogue wave and a pair of resonance stripe solitons to KP equation," Computers \& Mathematcs with Applications, vol. 76, no. 8, pp. 1938-1949, 2018.

[24] H. L. Wu and J. F. Song, "Mixed lump-stripe soliton solutions to a dimensionally reduced generalized Jimbo-Miwa equation," Applied Mathematics Letters, vol. 90, pp. 181-187, 2019.

[25] Y. H. Wang, H. Wang, H. H. Dong, H. S. Zhang, and C. Temuer, "Interaction solutions for a reduced extended (3+1)-dimensional Jimbo-Miwa equation," Nonlinear Dynamics, vol. 92, no. 2, pp. 487-497, 2018.

[26] H. Wang, "Lump and interaction solutions to the (2+1)dimensional Burgers equation," Applied Mathematics Letters, vol. 85, pp. 27-34, 2018.

[27] L. Chen, J. C. Chen, and Q. Y. Chen, "Mixed lump-soliton solutions to the two-dimensional Toda lattice equation via symbolic computation," Nonlinear Dynamics, vol. 96, no. 2, pp. 1531-1539, 2019.

[28] J. C. Chen, J. Lin, and S. Y. Lou, “Twisted lump, lumpoff and rogue wave of the $(2+1)$-dimensional Kaup-Kupershmidt equation," The European Physical Journal Plus, vol. 135, no. 3, p. 287, 2020.

[29] X. R. Hu, S. F. Shen, and Y. Y. Jin, "Rogue wave and interaction phenomenon to (1+1)-dimensional Ito equation," Applied Mathematics Letters, vol. 90, pp. 99-103, 2019.

[30] X. R. Hu, S. N. Lin, and L. Wang, "Integrability, multiple-cosh, lumps and lump-soliton solutions to a (2+1)-dimensional generalized breaking soliton equation," Communications in Nonlinear Science and Numerical Simulation, vol. 91, article 105447, 2020.

[31] M. D. Chen, X. Li, Y. Wang, and B. Li, “A pair of resonance stripe solitons and lump solutions to a reduced (3+1)-dimensional nonlinear evolution equation," Communications in Theoretical Physics, vol. 67, no. 6, p. 595, 2017.

[32] J. Wang, N. Xiong, and B. Li, "Peakon solutions of Alice-Bob -family equation and Novikov equation," Advances in Mathematical Physics, vol. 2019, Article ID 1519305, 8 pages, 2019.

[33] X. Li, Y. Wang, M. Chen, and B. Li, "Lump solutions and resonance stripe solitons to the $(2+1)$-dimensional SawadaKotera equation," Advances in Mathematical Physics, vol. 2017, Article ID 1743789, 6 pages, 2017.

[34] S. Yang, Z. Zhang, and B. Li, "Soliton molecules and some novel types of hybrid solutions to $(2+1)$-dimensional variable-coefficient Caudrey-Dodd-Gibbon-Kotera-Sawada equation," Advances in Mathematical Physics, vol. 2020, Article ID 2670710, 9 pages, 2020.

[35] B. Ren, W. X. Ma, and J. Yu, "Rational solutions and their interaction solutions of the $(2+1)$-dimensional modified dispersive water wave equation," Computers \& Mathematcs with Applications, vol. 77, pp. 2086-2095, 2019.

[36] B. Ren, W. X. Ma, and J. Yu, "Characteristics and interactions of solitary and lump waves of a (2+1)-dimensional coupled nonlinear partial differential equation," Nonlinear Dynamics, vol. 96, no. 1, pp. 717-727, 2019.
[37] S. Y. Lou and J. Lin, "Rogue waves in nonintegrable KdV-type systems," Chinese Physics Letters, vol. 35, no. 5, article 050202, 2018.

[38] F. Guo and J. Lin, "Interaction solutions between lump and stripe soliton to the (2+1)-dimensional Date-Jimbo-Kashiwara-Miwa equation," Nonlinear Dynamics, vol. 96, no. 2, pp. 1233-1241, 2019.

[39] Z. Zhang, Z. Qi, and B. Li, "Fusion and fission phenomena for (2+1)-dimensional fifth-order KdV system," Applied Mathematics Letters, vol. 116, article 107004, 2021.

[40] W. Q. Peng, S. F. Tian, L. Zou, and T. T. Zhang, "Characteristics of the solitary waves and lump waves with interaction phenomena in a $(2+1)$-dimensional generalized Caudrey-DoddGibbon-Kotera-Sawada equation," Nonlinear Dynamics, vol. 93, no. 4, pp. 1841-1851, 2018.

[41] H. Xu, Z. Y. Ma, J. X. Fei, and Q. Y. Zhu, "Novel characteristics of lump and lump-soliton interaction solutions to the generalized variable-coefficient Kadomtsev-Petviashvili equation," Nonlinear Dynamics, vol. 98, no. 1, pp. 551-560, 2019.

[42] W. Q. Peng, S. F. Tian, and T. T. Zhang, "Analysis on lump, lumpoff and rogue waves with predictability to the $(2+1)$ dimensional B-type Kadomtsev-Petviashvili equation," Physics Letters A, vol. 382, no. 38, pp. 2701-2708, 2018.

[43] Y. L. Ma and B. Q. Li, "Analytic rogue wave solutions for a generalized fourth-order Boussinesq equation in fluid mechanics," Mathematicsl Methods in the Applied Sciences, vol. 42, pp. 3942, 2019.

[44] B. Q. Li and Y. L. Ma, "Multiple-lump waves for a $(3+1)$ dimensional Boiti-Leon-Manna-Pempinelli equation arising from incompressible fluid," Computers \& Mathematics with Applications, vol. 76, no. 1, pp. 204-214, 2018.

[45] B. Q. Li and Y. L. Ma, "Interaction dynamics of hybrid solitons and breathers for extended generalization of Vakhnenko equation," Nonlinear Dynamics, vol. 102, no. 3, pp. 1787-1799, 2020.

[46] B. Q. Li, "Loop-like kink breather and its transition phenomena for the Vakhnenko equation arising from high-frequency wave propagation in electromagnetic physics," Applied Mathematics Letters, vol. 112, article 106822, 2021.

[47] B. Q. Li and Y. L. Ma, "Lax pair, Darboux transformation and Nth-order rogue wave solutions for a $(2+1)$-dimensional Heisenberg ferromagnetic spin chain equation," Computers \& Mathematics with Applications, vol. 77, no. 2, pp. 514-524, 2019.

[48] Y. L. Ma, "Interaction and energy transition between the breather and rogue wave for a generalized nonlinear Schrödinger system with two higher-order dispersion operators in optical fibers," Nonlinear Dynamics, vol. 97, no. 1, pp. 95-105, 2019.

[49] B. Q. Li and Y. L. Ma, "N-order rogue waves and their novel colliding dynamics for a transient stimulated Raman scattering system arising from nonlinear optics," Nonlinear Dynamics, vol. 101, no. 4, pp. 2449-2461, 2020.

[50] B. Q. Li and Y. L. Ma, "Extended generalized Darboux transformation to hybrid rogue wave and breather solutions for a nonlinear Schrodinger equation," Mathematics of Computation, vol. 386, article 125469, 2020.

[51] J. Matsukidaira, J. Satsuma, and W. Strampp, "Soliton equations expressed by trilinear forms and their solutions," Physics Letters A, vol. 147 , no. 8-9, pp. 467-471, 1990. 
[52] W. X. Ma, "Trilinear equations, Bell polynomials, and resonant solutions," Frontiers of Mathematics in China, vol. 8, no. 5, pp. 1139-1156, 2013.

[53] L. T. Gai, W. X. Ma, and M. C. Li, "Lump-type solution and breather lump-kink interaction phenomena to a $(3+1)$-dimensional GBK equation based on trilinear form," Nonlinear Dynamics, vol. 100, no. 3, pp. 2715-2727, 2020.

[54] R. Hirota, The Direct Method in Soliton Theory, Cambridge University Press, New York, NY, USA, 2004.

[55] Y. L. Ma and B. Q. Li, "Mixed lump and soliton solutions for a generalized (3+1)-dimensional Kadomtsev-Petviashvili equation," AIMS Mathematics, vol. 5, no. 2, pp. 1162-1176, 2020.

[56] Y. L. Ma and B. Q. Li, "Interactions between soliton and rogue wave for a (2+1)-dimensional generalized breaking soliton system: hidden rogue wave and hidden soliton," Computers \& Mathematcs with Applications, vol. 78, no. 3, pp. 827-839, 2019. 